\title{
MAPPING OF BLUE CARBON ECOSYSTEMS: EFFECT OF PROXIMITY, ACTIVITY TYPES AND FREQUENCY OF VISITS IN THE ACCURACY OF PARTICIPATORY MAPS
}

\author{
R.R.T. Francisco ${ }^{1,2}$, A.C. Blanco ${ }^{1,2}$, M.A.G. Manalilii ${ }^{1,2}$, N.B. Gatdula ${ }^{1,2}$, \\ A.J.G. Songcuan ${ }^{1}$, K.P.C. Landicho ${ }^{1,2}$, R.B. Reyes ${ }^{1,2}$, R.J.A. Apura ${ }^{1,2}$ \\ ${ }^{1}$ Training Center for Applied Geodesy and Photogrammetry, University of the Philippines-Diliman, Quezon City, Philippines 1101 \\ ${ }^{2}$ Department of Geodetic Engineering, College of Engineering, University of the Philippines-Diliman, Quezon City, Philippines \\ 1101 \\ (rtfrancisco, acblanco, nbgatdula, kclandicho, raapura)@up.edu.ph; \\ (mikelmanalili21, ajsongcuan)@gmail.com; rbr_lally@yahoo.com
}

Commission VI, WG VI/4

KEY WORDS: Mangroves, Seagrasses, Participatory Mapping, Geographic Information System

\begin{abstract}
:
Interest in blue carbon has drastically increased in recent years, particularly in improving the coastal resource carbon storage estimates and the development of methodology for identifying and monitoring such resources. In coastal resource mapping, participatory mapping techniques have the potential to provide a level of granularity and detail by taking advantage of local knowledge. In this work, we aim to evaluate the agreement between blue carbon ecosystem status obtained from participatory mapping versus the ones discriminated from satellite images, as well as assess how "relative proximity" to landmarks affects the accuracy. Results showed that the accuracy of mapped mangrove extents, evaluated as intersection-over-union, is positively correlated with frequency of visits. It is also found that maps generated by participants who have jobs or activities that nurture awareness about mangroves and seagrasses tend to agree better with remotely-sensed maps. The participants were even able to identify small patches of mangroves and seagrasses that are not present in the classified satellite images. While our initial work explores factors that impact the consistency between these sources, there is a need to establish a baseline for which both sources of information are evaluated against; and define relevant accuracy metrics. Our final goal is to systematically combine these two sources of information for an accurate holistic coastal resource map.
\end{abstract}

\section{INTRODUCTION}

The oceans encompass an estimated 38,000 gigatons (Gt) of carbon and has absorbed a third of the anthropogenic carbon dioxide $\left(\mathrm{CO}_{2}\right)$ emission through physical, chemical and biological processes (Mcleod et al., 2011). Several research studies have emphasized that coastal ecosystem composed of plants such as mangroves and seagrasses play a valuable role in the sequestration of carbon. While these coastal plants are only a smaller percentage compared to the overall global forests, mangrove forests account for $13.5 \mathrm{Gt}$ per year of carbon sequestration (Alongi, 2012) and seagrass meadows, which occupies only $0.2 \%$ of global ocean account for 27.4 Gt per year (Duarte et al. 2005) - far more efficient than terrestrial forests. With an estimated 0.15-1.02 Petagram of carbon $(\mathrm{Pg})$ emissions annually (Pendleton et al., 2012; Miteva et al., 2015), accurately accounting for the $\mathrm{C}$ uptake of coastal ecosystems becomes critical.

Blue carbon (carbon stored in oceans, including coastal marine environments) study has increased in recent years, more primarily in improving the estimates of coastal resource blue carbon storage (Lavery et al., 2013). This also includes the development of workflows in identifying and monitoring blue carbon resources (e.g. mangroves) as well as estimating the corresponding carbon capacity. Remote sensing through satellite imagery, i.e., optical and radar (Purnamasayangsukasih et al., 2016) is largely considered as a viable method by far (Mensah, 2013) as it offers larger coverage compared to traditional data collection approaches. In coastal resource mapping where species-level classification is of interest, satellite-based techniques often suffer from temporal and spatial inconsistency (Duffy et al., 2018) and spatial resolution of available satellite images are still coarse for such level of mapping. In this context, detailed information from participatory mapping, which takes advantage of local knowledge, can help address such limitations.

Participatory mapping follows the same fundamental principles and objectives as participatory research; but combines the informal knowledge of the local community with modern cartography tools to create composite maps. This approach allows the locals to create maps based on bits of qualitative, quantitative, tangible and/or intangible information around a given area developed over time through their personal experiences (Warner, 2015). The goal of participatory mapping is to incorporate local spatial and environmental knowledge of spaces to formal forms of geographic data in order to identify, understand, analyze and resolve issues relating to such spaces (Aheto et al., 2016; Beverly et al, 2008; Cadag and Gaillard, 2012).

Knowledge of the coastal communities are vital in filling in the gaps where remotely-sensed data is sparse or limited. Several studies have shown that such information is much richer and granular; and provides holistic and long-term accounts based on experience relating to the pertinent coastal resources (Mensah, 2013; Brown, 2018). For instance, the community's first-hand experience can aid in the identification of various coastal plant species; and historical accounts can shed insights on the factors that led to resource growth or degradation. However, this poses the challenge of ensuring its accuracy and reliability as community-based mapping activities tend to depend on 
participants' memory and are inherently subjective (Vajjhalla, 2005). Precision of object and spatial memory can be driven by the familiarity of the participant over his/her environment and proximity to recognizable landmarks. This impacts the analysis of blue carbon ecosystems, corresponding blue carbon stocks, future conservation and rehabilitation strategies.

To date factors influencing the accuracy of a community-based map have not been rigorously studied. With respect to blue carbon ecosystems, identifying these factors and quantifying their impact on the quality of generated maps is an important step to help guide subsequent mangrove/seagrass mapping activities (i.e. employing the right people to participate).

In this study, we aim to evaluate the effect of relative proximity of landmarks or places frequented by locals to blue carbon ecosystem information generated through participatory mapping as compared to satellite-derived classified maps. Proximity in this case means Euclidean proximity in space that tend to be influenced by frequency of visits to places as well as seasonal activities in the area. This research is part of Project 10: Geospatial Decision Support Systems and Capacity Building on Geomatics for Mangrove Seagrass Conservation (CapGeoDSS) under the Integrated Assessment and Modelling of Blue Carbon Ecosystems for Conservation and Adaptive Management (IAMBlueCECAM) Program funded by the Department of Science and Technology Philippine Council for Industry, Energy and Emerging Technology Research and Development (DOSTPCIEERD).

Our contribution is summarized as follows:

- We compare the agreement between community-based versus remotely-sensed mangrove and seagrass extents ( $\mathrm{Sec}$. 3.1)

- We demonstrate that participants' proximity and familiarity affect the accuracy of a community-based mangrove and seagrass maps.

- We show that daily activities of the participants (e.g. fishing) is an important criterion for choosing the right people in a mangrove/seagrass mapping activity.

\section{METHODOLOGY}

\subsection{Study Area}

Busuanga is an island municipality located in the north-eastern section of Palawan, Philippines - an archipelagic province known for its pristine marine environmental condition and several wildlife sanctuary and protected areas. The study area is composed of the three coastal barangays in Busuanga namely Concepcion, Bogtong and Salvacion (Fig. 1a). Smaller islands and marine protected area with mangroves and seagrass beds are within these coastal sites.
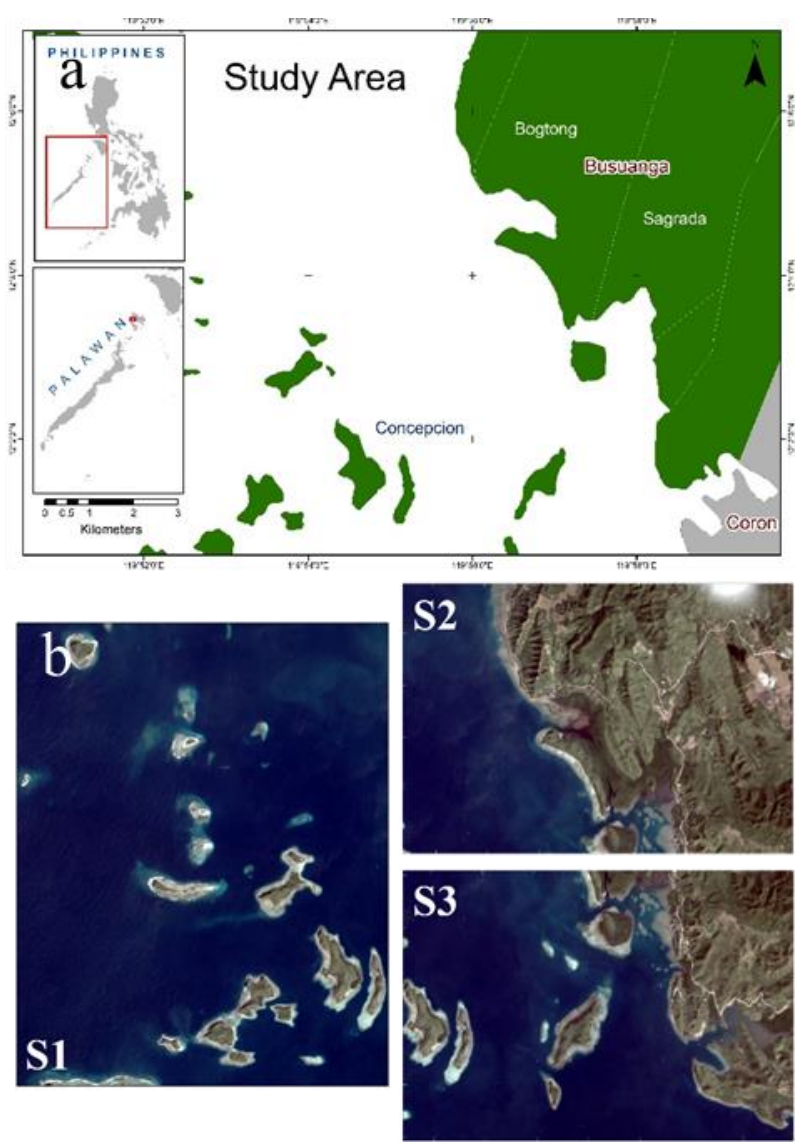

Figure 1. (a) The study area located in Busuanga, Palawan, Philippines. The site is known for marine biodiversity; and (b) The PlanetScope image utilized for the site. The site was divided into three sections labelled S1, S2, S3. Each section has a small overlap among other sections Each map was assigned to a group of volunteers in the participatory mapping activity.

\subsection{Participatory Mapping Workflow}

The research workflow, shown in Fig. 2, is divided into three stages, namely, pre-mapping, mapping proper and post-mapping.

\subsubsection{Pre-mapping Stage}

In the pre-mapping stage, the base map of the study area was prepared using a 3-meter resolution PlanetScope image (Planet Team, 2017) of Busuanga, Palawan. This base map was further divided into three sections for more detailed and manageable mapping of information (see Fig. 1b).

Participants from different sectors of the community, namely, elders, fisherfolks, students, women and locals who are residents for more than 10 years were requested to attend the activity. A survey questionnaire was also prepared to capture relevant information such as demography, participant's familiarity in mapping, environment, community, and feedback on the mapping activity. 


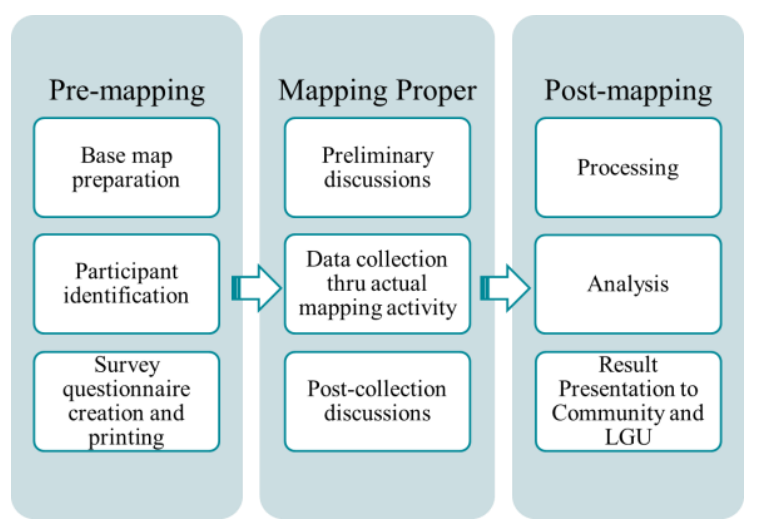

Figure 2. Participatory mapping workflow in the study. The workflow is divided into three stages: pre-mapping, mapping proper and post-mapping.

\subsubsection{Mapping Proper}

A participatory mapping activity, attended by 24 residents from the three coastal sites, was facilitated in Busuanga, Palawan on May 29 to 30, 2018. Preliminary details such as the overview of the activity and mapping instructions were presented to the participants. They were then provided with the survey questionnaires that they need to answer fully. The participants were divided into three groups (Table 1) and each group was given one section of the whole site. They were asked to identify and map the extent per species of mangroves and seagrasses present in their area. They also mapped the extents where coastal resources are present as well as the uses, issues and problems and past damages in these resources. Individual mapping of places that they visited was also done together with the identification of these activities and frequency of visit. After the activity, each group chose a representative and presented their resulting map to the other participants. They also answered the survey questionnaire during and after the mapping proper.

\begin{tabular}{|c|c|l|}
\hline Group & Age & Job/Work \\
\hline 1 & 18 & None \\
& 42 & Nutrition scholar \\
& 54 & MPA Guard \\
& 44 & None \\
& 29 & Health worker \\
& 19 & None \\
& 47 & Health worker \\
\hline 2 & 42 & IPMR \\
& 49 & MPA Guard \\
& 43 & Mangrove planter \\
& 55 & Mangrove planter \\
& 46 & Fisherman \\
& 45 & Health worker \\
& 49 & Health worker \\
& 34 & Health worker \\
& 60 & Health worker \\
& 50 & Brgy. Official \\
\hline 3 & 67 & Fisherman \\
& 64 & Fisherman \\
& 24 & Brgy. Official \\
& 42 & Businessman \\
& 55 & Fisherman \\
& 40 & Fisherman \\
& 51 & Fisherman \\
\hline
\end{tabular}

Table 1. Assigned groups of the 24 residents of Busuanga who volunteered for the participatory mapping activity.

\subsubsection{Post-Mapping Stage}

For the post-mapping activity, the participatory maps were scanned, digitized and analysed. The result of the activity was compared with the satellite-based mangrove and seagrass extent maps produced by IAMBlueCECAM Program's Project 1: Mangrove Remote Sensing Using LiDAR, Radar, Multispectral and Hyperspectral Data (MaRS) and Project 2: LiDAR, Hyperspectral, and Sonar Remote Sensing of Seagrass Meadows (SeaRS). The mangrove and seagrass maps were derived from Sentinel 2 and PlanetScope images of the study area, respectively. These blue carbon ecosystems were then classified using the supervised maximum likelihood classification method. A presentation to and consultation with the locals and the municipal government of Busuanga on the results of the study were also conducted.

\section{RESULTS AND DISCUSSIONS}

\subsection{General observations}

There was a total of 20 species of mangroves in 95 areas identified by Group 1 (G1), 33 species in 63 areas by Group 2 (G2), and 13 species in 93 areas were mapped by Group 3 (G3). Common mangrove families mapped were Avicennia, Bruguiera and Rhizophora. For seagrass, G1, G2, and G3 respectively identified 7 species in 34 areas, 7 species in 12 areas, and 3 speciesn 12 areas. Halodule, Cymodoceae, and Enhalus were the common seagrasses observed.

Overall, the participants identified 353.67 (361.50) hectares of mangroves (seagrass) within the study area. Comparing these estimates with mangrove and seagrass maps using satellite images (Fig. 3), initial visual inspection indicated that the extents (or patches of) the marine resources were closely captured by the participants. However, it was also observed that there are instances where resources were identified in the participatory maps (PM) but not in the satellite-based maps; and vice versa. In Fig. 3a for instance, a large portion of mangrove forests in the south of Bogtong present in the satellite map was not delineated by the participants as mangrove. On the other hand, we also found certain regions particularly in the western islands of Concepcion that were identified by the locals as seagrass (Fig. 3b). Such features were not present in the satellite-based classification map.

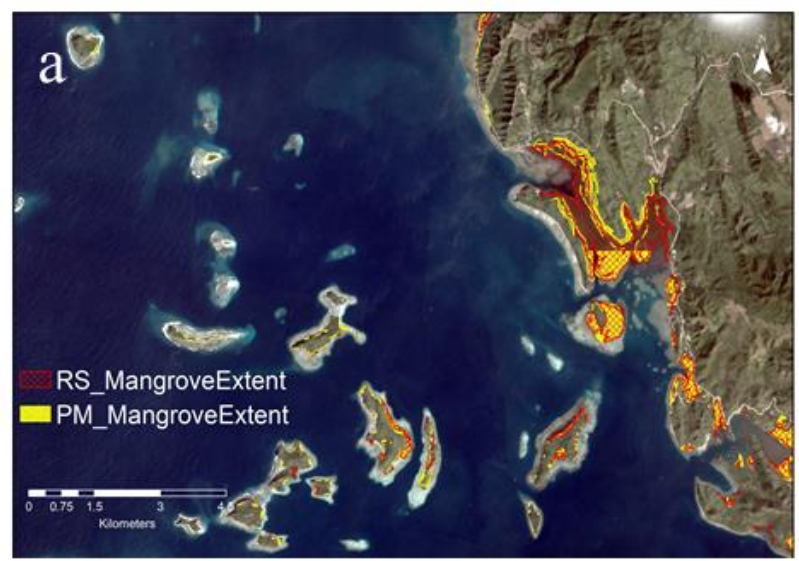




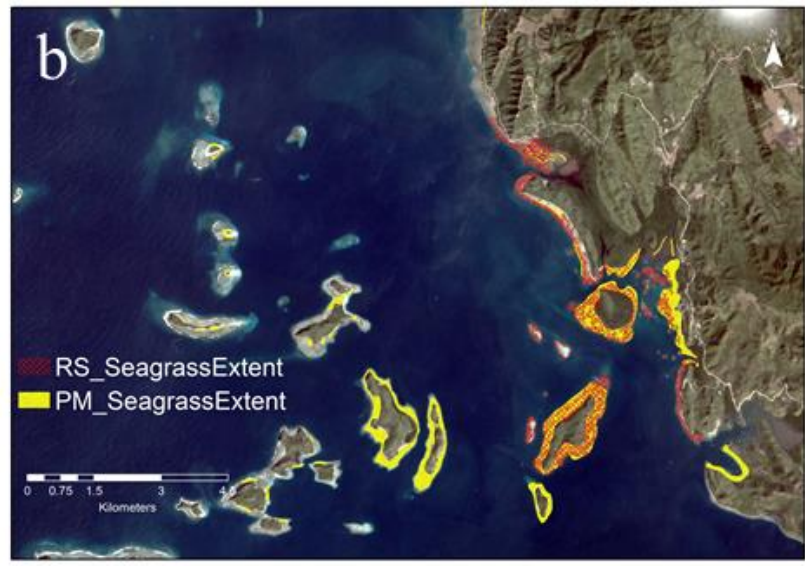

Figure 3. (a) Overlayed patches of mangroves obtained from participatory map (PM, yellow solid) and Sentinel-2 classified image (RS, red hatched); (b) Overlayed patches of seagrass meadows obtained from participatory map (yellow solid) PlanetScope classified image (red hatched).

\begin{tabular}{|c|c|c|c|c|}
\hline \multirow{2}{*}{ Source } & \multicolumn{4}{|c|}{ Area in hectares } \\
\hline & G1 & $\mathrm{G} 2$ & G3 & Overall \\
\hline PM & 26.56 & 79.65 & 247.47 & 353.67 \\
\hline Satellite & 12.33 & 309.50 & 205.07 & 460.83 \\
\hline Intersection & 4.26 & 30.82 & 183.93 & 219.01 \\
\hline Union & 34.62 & 358.33 & 268.61 & 603.47 \\
\hline IoU $(\%)$ & 12.31 & 8.60 & 68.41 & 36.29 \\
\hline PM & - & 6.86 & 354.63 & 361.50 \\
\hline Satellite & -- & 117.25 & 196.93 & 252.00 \\
\hline Intersection & -- & 2.06 & 124.93 & 126.99 \\
\hline Union & -- & 122.06 & 426.63 & 486.50 \\
\hline IoU (\%) & -- & 1.69 & 29.28 & 26.10 \\
\hline
\end{tabular}

Table 2. Intersection-over-union (IoU) between participatory maps (PM) and satellite-based classified maps of mangroves and seagrass.

To quantify the agreement between the satellite-derived and resulting participatory maps, the spatial intersection-over-union (IoU) for both mangrove and seagrass maps were calculated (Table 2). In the combined mangrove forest area (union) of 603.47 ha, both maps only agreed on 219.01 ha (36.29\%) of mangroves. The agreement between maps for seagrass was $26.10 \%$. G3 generated the highest value among the three for mangrove extent with $68.41 \%$ agreement. For G1, the mangrove area classified (27.56 ha) was twice that of the satellite-based map (12.33 ha) and only 4.26-ha intersection zone. This resulted to $12.31 \%$ agreement only. G2 has the lowest value of IoU $(8.60 \%)$ that was brought about the misclassification of a large parcel of mangrove forest in the south of Bogtong. For seagrass, the portion for G1 was not covered by the satellite-derived map by SeaRS, thus no IOU was computed. Similar to the mangrove maps, G3 participants produced a result with higher agreement to the satellite-based seagrass map compared to G2.

\subsection{Effect of "proximity" and frequency of visits on map accuracy}

We hypothesize that the agreement between PM and satellitederived map is linked to the familiarity of the participant to the location being mapped; that is, the "proximity", based on distance, by which an observer can perceive his surroundings and frequency of his visits. In Fig. 4, the frequency of visits of all participants in various locations in the map is plotted. The mainland (east side) is more visited more often than the islands in the west section. Participants who visit the islands were mostly fishermen (not shown in the figure). Therefore, it is expected the accuracy of the PM would be relatively higher in the mainland due to close proximity and higher frequency of visits.

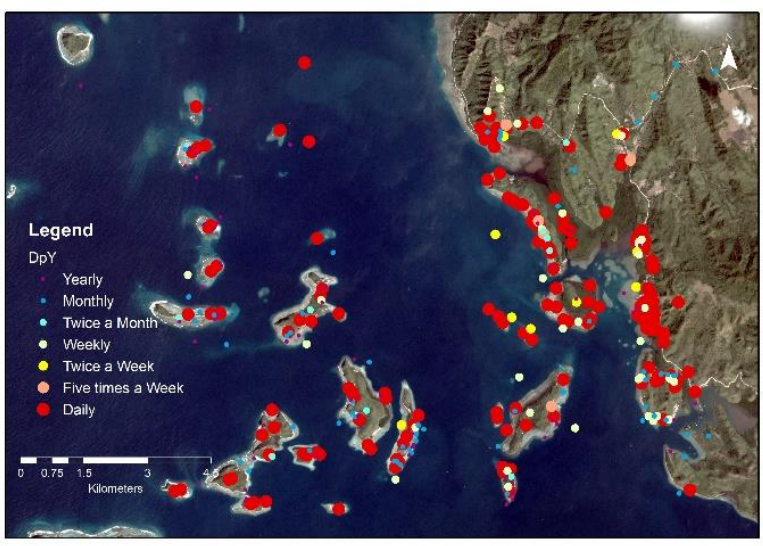

Figure 4. Frequently visited locations of all participants. The mainland (east side) is frequently visited while only a few of the participants visit the islands (west side).

To investigate this, the map was divided into $12 \times 18$ (row $\times$ columns) $1 \times 1 \mathrm{~km}$ grids. For each $g(z)$, the IoU between PM and satellite-based maps were computed and correlated with the aggregate visits per year in the grid given by:

$$
V_{g(z)}=\sum_{p} v(p) \text { if } \operatorname{buffer}(p, r) \cap g(z)>0
$$

where $\quad z=$ grid index

$p=$ location point

$v(p)=$ visits per year at point $p$

$r=$ buffer radius; set to 500 meters.

Here, the $v(p)$ was only added to $V_{g(z)}$ if a point with a $500-\mathrm{m}$ buffer radius $r$ intersected with $g(z)$. The calculations were done for each group in both mangrove and seagrass maps. Correlation between IoU and $V_{g(z)}$ was then obtained using:

$$
\text { Pearson's } r=\frac{n\left(\sum x y\right)-\left(\sum x\right)\left(\sum y\right)}{\sqrt{\left[n \sum x^{2}-\left(\sum x\right)^{2}\right]\left[n \sum y^{2}-\left(\sum y\right)^{2}\right]}}
$$

where $x$ and $y$ are the independent $\left(V_{g(z)}\right)$ and dependent (IoU) variables, respectively. In order not to introduce an unnecessary bias, data points for grids with zero-valued IoU and $V_{g(z)}$ were removed from the analysis.

For mangroves (Fig. 5a), IoU is generally positively correlated with $\mathrm{V}$, which suggests that degree of familiarity to a location plays a role in producing reliable participatory resource maps. G3 generated the largest correlation coefficient $r=0.38$. G2 and G1 produce 0.35 and -0.21 , respectively, but these results cannot support nor contradict our inference for G3 due to lack of data points. For G1, producing a non-zero IoU was particularly challenging partly because of the limited sizes of mangrove patches in the area.

For seagrass maps, the negative correlation for G3 (-0.71) seem to imply that familiarity diminishes map accuracy. Inspection of the scatter plot showed two prominent clusters: (1) very high IoU achieved with $V_{g(z)}<2000$; and (2) low IoU even when $V_{g(z)}>$ 3000 . In the latter, the grid points z-116, z-136 and z-156 are part 
of the vertical coastline covering west of mainland Concepcion and south Sagrada. In these locations, the participants identified significantly wider seagrass meadows compared to that of derived from PlanetScope images resulting to low IoUs. Nevertheless, both clusters show positive correlation between IoU and $V_{g(z)}$ - consistent with the findings for mangroves. G2 also demonstrated the positive correlation, there is a need for more data to arrive at a conclusive result. No data were found for G1 as no intersection between PM and satellite-based seagrass maps.
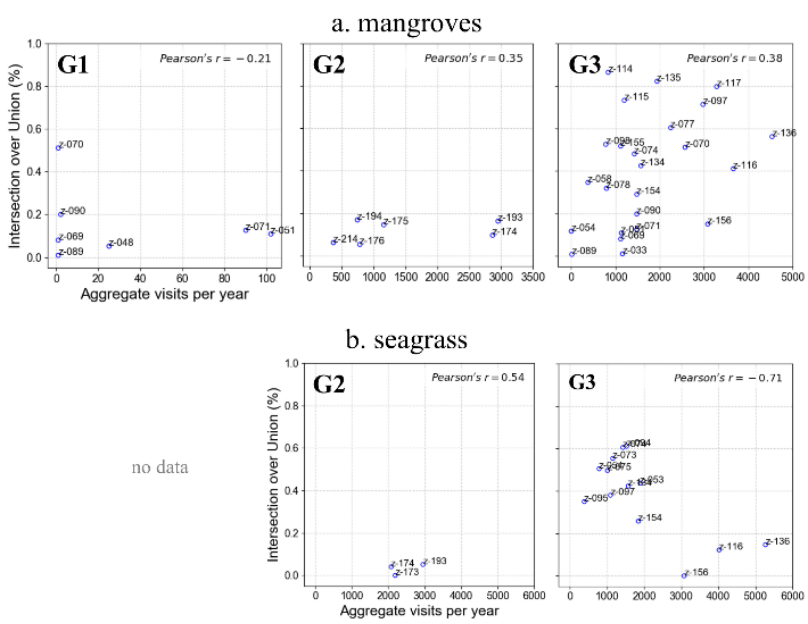

Figure 5. IoU vs. aggregated visits per year $V_{g(z)}$ for (a) mangroves and (b) seagrass. Data points for grids with zerovalued IoU and $V_{g(z)}$ were not included in the plots.

\subsection{Influence of the participants' daily activities and other demographic information}

Maps generated by participants who have jobs or activities that nurture awareness about mangroves and seagrass tend to agree better with remotely-sensed maps. G3 which was composed of 4 fishermen, 1 MPA guard and 1 barangay official resulted to $68.48 \%$ accuracy of mapped mangrove extents relative to the satellite-based map. On the other hand, G1, which was composed of 3 health workers, 1 nutrition scholar, 3 unemployed individuals, and only 1 MPA guard, was not able to delineate the extents as accurately. The difficulty can be further attributed to the characteristics of map assigned to them (S1) - smaller islands far away from the mainland that were frequently visited almost exclusively by fishermen. G2 comprised of an ample number of MPA guards, mangrove planters and fishermen; however, misclassification prevented the group from generating high IoU resource maps.

Further analysis revealed that participants do not necessarily need to be adept in creating a map. Applying multiple correspondence analysis (MCA) on demographic information such as mapping abilities with respect to job and education level of the participants showed that the ability/inability to create a map is not indicative of one's credibility. In Fig. 6, the job "fisherman" is closely related to "Can make a map? - $N$ " but it turned out that G3, composed mostly of fishermen, generated remarkably highquality maps. At the very least, the ability to read a map is necessary as creating a map can be taught and assessed before the actual mapping begins.

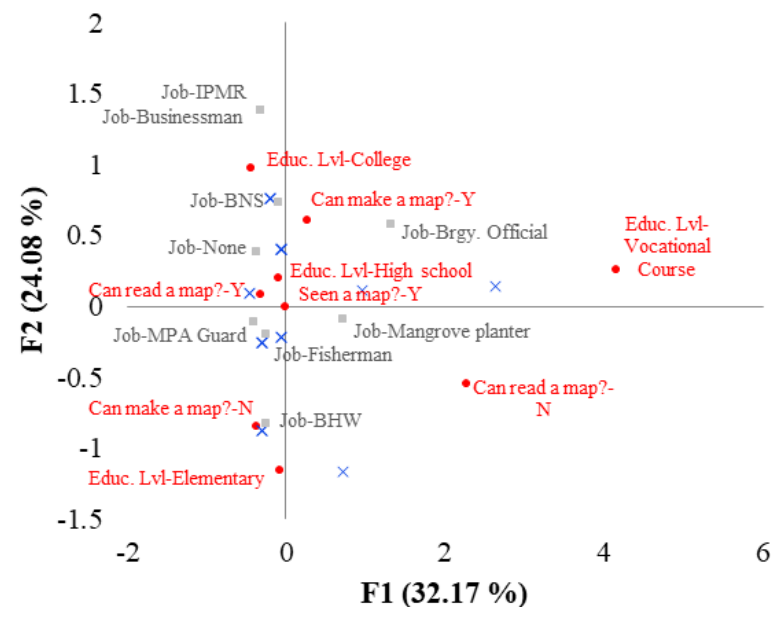

Figure 6. Symmetric plot of the first two principal coordinates relating the participants' job, education level and mapping abilities. The ' $\mathrm{Y}$ ' ('N') after each question indicates yes (no).

Our results have clearly demonstrated another critical insight in participatory mapping that the job or the daily activities of the participants is an important criterion for choosing the right people in a mapping activity. In the case of this marine resource mapping study, fishermen and MPA guards contributed substantially to the accuracy of the maps.

\section{FINAL REMARKS}

A subtle limitation of our work is that the results presented in Sec. 3 came from a single mapping activity only; and consolidating data from multiple mapping activities may indeed yield more solid results. However, these initial findings reveal promising insights. By measuring the agreement of PM and satellite-based maps, we have established a baseline grasp of the capability of participatory techniques in creating reliable resource maps. We have shown that proximity, familiarity with a location, and type of activity influence the accuracy of participatory maps. We recommend that these criteria to be factored in determining the most qualified locals in a resource mapping activity.

Maps of mangroves and seagrass (or generally any natural resource) produced through participatory techniques need to be carefully assessed for it to be useful for decision-making and planning. We have done an initial quantitative assessment. However, since maps generated through classification from satellite images cannot be considered as ground truth, field validation on the entire study area is integral to truly assess the correctness of PM. The same analysis can be performed using the data from field surveys to confirm our findings in Sec. 3 .

Locals were able to identify certain regions of mangroves /seagrasses that were not captured by satellite image classification; but we cannot dismiss the power of satellitederived maps either. Characterizing the strengths and weakness of both sources using field survey data as reference (e.g. identifying scenarios where PM is more reliable than satellitebased classification and vice-versa) is an important step towards developing a standard mapping workflow/algorithm for integrating local knowledge with resource maps produced with $3 \mathrm{~S}$ (i.e., remote sensing, geographic information, global navigation satellite systems) technology. 
Ultimately, an accurate depiction of the status of blue carbon ecosystem by considering all possible sources of information leads to a better estimate of $\mathrm{CO}_{2}$ sequestration; and therefore, better planning and conservation strategies. An accurate map of marine resources is fundamental. Factoring in species information, which was obtained from locals, is the next step. As a final note, the survey questionnaires administered to the participants during the mapping activity contain ancillary information such as knowledge on the changes in the extent of their marine resources over time, as well as reasons for such changes that will be essential for future analysis.

\section{ACKNOWLEDGEMENTS}

This work was primarily funded by the Department of Science and Technology Philippine Council for Industry, Energy and Emerging Technology Research and Development (DOSTPCIEERD) with Project Nos. 04038, 04040 and 04048, 2017. The researchers would also like to acknowledge the support of the University of the Philippines Training Center for Applied Geodesy and Photogrammetry (UP TCAGP), the JICA-JST BlueCARES Project, and the active participation of Barangays Concepcion, Sagrada and Bogtong and the local government of Busuanga, Palawan.

\section{REFERENCES}

Aheto, D.W., Kankam, S., Okyere, I., Mensah, E., Osman, A., Jonah, F.E., Mensah, J.C., 2016. Community-based mangrove forest management: Implications for local livelihoods and coastal resource conservation along the Volta estuary catchment area of Ghana, Ocean and Coastal Management, 127, pp. 43 - 54.

Alongi, D.M., 2012. Carbon sequestration in mangrove forests, Carbon Management, 3(3), pp. 313 - 322.

Beverly, J.L., Uto, K., Wilkes, J., and Bothwell, P., 2008. Assessing spatial attributes of forest landscape values: an internet-based participatory mapping approach, Can. J. For. Res., 38 , pp. $289-303$.

Brown, M.I., Pearce, T., Leon J., Sidle, R., and Wilson, R., 2018. Using remote sensing and traditional ecological knowledge (TEK) to understand mangrove change on the Maroochy River, Queensland, Australia, Applied Geography, 94, pp. 71 - 83.

Cadag, J.R.D., and Gaillard, J.C., 2012. Integrating knowledge and actions in disaster risk reduction: the contribution of participatory mapping, Area, 44(1), pp. $100-109$.

Duarte, C.M., Middelburg, J.J., Caraco, N., 2005. Major role of marine vegetation on the oceanic carbon cycle. Biogeosciences, 2 , pp. $1-8$.

Duffy, J.P., Pratt, L., Anderson, K., Land, P.E., and Shutler, J.D., 2018. Spatial assessment of intertidal seagrass meadows using optical imaging systems and a lightweight drone. Estuarine, Coastal and Shelf Science, 200, pp. $169-180$.

Lavery, P.S., Mateo, M.Á., Serrano, O., and Rozaimi, M., 2013. Variability in the carbon storage of seagrass habitats and its implications for global estimates of blue carbon ecosystem service. PloS One, 8(9), e73748.
Mcleod, E., Chmura, G.L., Bouillon, S., Salm, R., and coauthors, 2011. A blueprint for blue carbon: toward an improved understanding of the role of vegetated coastal habitats in sequestering $\mathrm{CO} 2$. Frontiers in Ecology and the Environment, 9(10), pp. 552-560.

Mensah, J., 2013. Remote sensing application for mangrove mapping in the Ellembelle district in Ghana. USAID Integrated Coastal and Fisheries Governance Program for the Western Region of Ghana. Narragansett, RI: Coastal Resources Center, Graduate School of Oceanography, University of Rhode Island.

Miteva, D.A., Murray, B.C., and Pattanayak, S.K., 2015. Do protected areas reduce blue carbon emissions? A quasiexperimental evaluation of mangroves in Indonesia, Ecological Economics, 119, pp. 127 - 135.

Pendleton, L., Donato, D.C., Murray, B.C., Crooks, S., and coauthors, 2012, Estimating global "blue carbon" emissions from conversion and degradation of vegetated coastal ecosystems, PloS One, 7(9), e43542.

Planet Team, 2017. Planet Application Program Interface: In Space for Life on Earth. San Francisco, CA. https://api.planet.com.

Purnamasayangsukasih, P.R., Norizah, K., Ismail, A.A., and Shamsudin, I. (2016, June). A review of uses of satellite imagery in monitoring mangrove forests. In IOP Conference Series: Earth and Environmental Science, Vol. 37, No. 1, p. 012034.

Vajjhalla, S.P., 2005. Integrating GIS and Participatory Mapping in Community Development Planning. ESRI International User Conference, Sustainable Development and Humanitarian Affairs Track, San Diego, CA

Warner, C., 2015. Participatory Mapping: a literature review of community-based research and participatory planning. Social Hub for Community and Housing Faculty of Architecture and Town Planning Techion. 